\title{
Fast Solvers for Queueing Systems with Negative Customers *
}

\author{
You-Wei Wen* ${ }^{*}$, Wai-Ki Ching, ${ }^{*}, \quad$ Michael K. $\mathrm{Ng}^{b}$
}

12 March 2006

\begin{abstract}
In this paper, we are interested in solving Markovian queueing systems having Poisson batch arrivals, exponential servers and negative customers. Preconditioned conjugate gradient method is applied to solving the steady-state probability distribution of queueing system. Preconditioner were constructed by exploiting near-Toeplitz structure of the generator matrix and the Gohberg-Semumcul formula. We proved the preconditioned system has its spectrum clustered around one. Numerical results are given to demonstrate the efficient of our method.
\end{abstract}

Key Words. Queueing system, Negative Customer, Gohberg-Semencul formula, Preconditioned Conjugate Gradient method, Preconditioners.

\section{Introduction}

In this paper, we are interested in solving the steady-state probability distribution of queueing systems having Poisson batch arrivals, exponential servers and negative customers. The idea of negative customer was first discussed by Gelenbe in the modelling of neural networks $[4,5]$. To understand the role of a negative customer we consider the following examples. One may consider a communication network in which messages are transmitted in a packet-switching mode. An arrival of a negative customer represents a server fails during a transmission. Therefore part of the messages will be lost. Another example, in a manufacturing system where a negative arrival represents a cancellation of a job. These lead to many applications in the modelling of manufacturing systems, telecommunication systems and many others such as reliability models [8]. Theoretical results including the stability analysis has been discussed by Gelenbe et al. $[4,5,6]$.

*The Advanced Modeling and Applied Computing Laboratory and Department of Mathematics, The University of Hong Kong, Pokfulam Road, Hong Kong. (Email: wkc@maths.hku.hk) Research supported in part by RGC Grants, HKU CRCG Grants and Hung Hing Ying Physical Sciences Research Fund. ${ }^{b}$ Department of Mathematics, The Hong Kong Baptist University, Kowloon Tong, Hong Kong. 
Here we consider the queueing system studied by Ching [2]. The input of the queueing system is assumed to be an exogenous Poisson batch arrival process with mean batch interarrival time $\lambda^{-1}$. The batch size of each arrival is assumed to follow a given discrete probability distribution $a_{i}, i=1,2, \ldots$, . where $\lambda_{i}=a_{i} \lambda$ for $i \geq 1$ and $a_{i}$ is the probability that the batch size is $i$. We note that $\lambda_{i}$ is the batch arrival rate for a batch of size $i$ and we have

$$
\lambda=\sum_{k=1}^{\infty} \lambda_{k}
$$

In the queueing model, it is assumed that there are $s$ identical independent exponential servers of mean service rate $\mu$. The queueing discipline is First-Come-First-Served (FCFS) and it is a block customer clear as there is only a waiting space of size $(n-s-1)$. Arrived customers (tasks) will be cleared from the system when the waiting space is full. Moreover, if the arrival batch size is larger than the waiting place left, then only part of the arrival batch will be accepted, the other customers will be treated as overflows and will be cleared from the system. For the arrival process of negative customers, it is also assumed as a Poisson process with mean rate $\tau$. The number of customers to be killed is assumed to follow a discrete probability distribution $b_{i}, i=1,2, \ldots$, . Here we assume that if the arrived negative customer is supposed to kill $i$ customers in the system and the number of customers in the system is less than $i$, then the queue becomes empty. The killing strategy is to remove the customers in the front of the queue. We let $\tau_{i}=b_{i} \tau$ where $b_{i}$ is the probability that the number of customers to be killed is $i$ and we have

$$
\tau=\sum_{k=1}^{\infty} \tau_{k}
$$

The generator matrix of the queueing system is given by

$$
\mathbf{H}_{n}=\left(\begin{array}{cccccccc}
\lambda & -u_{1} & -u_{2} & -u_{3} & \ldots & \ldots & \ldots & -u_{n-1} \\
-\lambda_{1} & \lambda+\tau+\mu & -2 \mu-\tau_{1} & -\tau_{2} & -\tau_{3} & \ldots & \ldots & -\tau_{n-1} \\
-\lambda_{2} & -\lambda_{1} & \lambda+\tau+2 \mu & \ddots & \ddots & \ddots & & \vdots \\
\vdots & -\lambda_{2} & \ddots & \ddots & -s \mu-\tau_{1} & -\tau_{2} & \ddots & \vdots \\
\vdots & \vdots & \ddots & \ddots & \lambda+\tau+s \mu & \ddots & \ddots & -\tau_{3} \\
\vdots & \vdots & \ddots & \ddots & \ddots & \ddots & \ddots & -\tau_{2} \\
-\lambda_{n-2} & -\lambda_{n-3} & -\lambda_{n-4} & \cdots & -\lambda_{2} & -\lambda_{1} & \lambda+\tau+s \mu & -s \mu-\tau_{1} \\
v_{1} & -v_{2} & -v_{3} & \cdots & \cdots & -v_{n-2} & -v_{n-1} & \tau+s \mu
\end{array}\right)
$$

where

$$
u_{1}=\tau, \quad u_{i}=\tau-\sum_{k=1}^{i-1} \tau_{k}, i=2,3, \ldots,
$$

and $v_{i}$ is defined such that the $i$ th column sum is zero. Clearly $\mathbf{H}_{n}$ has zero column sum, positive diagonal entries and non-positive off diagonal entries. From Perron and Frobenius theory [14, p. 30], $\mathbf{H}_{n}$ has a one-dimensional null-space with a positive null vector. Let $p_{i}$ be 
the stationary probability that there are $i$ customers in the system. The stationary probability vector $\mathbf{p}=\left(p_{1}, p_{2}, \ldots, p_{n}\right)^{t}$ of the queueing system is the normalized null-vector of the generator matrix $\mathbf{H}_{n}$. More precisely, $\mathbf{p}$ satisfies

$$
\mathbf{H}_{n} \mathbf{p}=\mathbf{0}, \quad p_{i} \geq 0 \text { and } \sum_{i=1}^{n} p_{i}=1 .
$$

We note that $\mathbf{H}_{n}$ has a one-dimensional null-space, the probability vector $\mathbf{p}$ can then be solved by considering the following linear system (see $[2,3])$ :

$$
\mathbf{Q}_{n} \mathbf{x}=\left(\mathbf{H}_{n}+\mathbf{e}_{n} \mathbf{e}_{n}^{t}\right) \mathbf{x}=\mathbf{e}_{n}
$$

where $\mathbf{e}_{n}=(0,0, \ldots, 0,1)^{t}$ is an unit vector. The probability distribution vector $\mathbf{p}$ is obtained by normalizing $\mathbf{x}$. Thus let us concentrate on solving the non-homogeneous systems of the form

$$
\mathbf{Q}_{n} \mathbf{X}=\mathbf{e}_{n}
$$

We remark that $\mathbf{Q}_{n}^{t}$ is an irreducibly diagonally dominant matrix, therefore if the system (2) is solved by classical iterative methods such as the Jacobi or the Gauss-Seidel methods, both methods will be convergent for all initial guesses.

Many useful system performance indicators of the queueing system (the blocking probability of the system and the expected number of customers in the system) can be written down in terms of the probability distribution vector $\mathbf{p}$. Therefore fast numerical algorithms for solving the queue systems are essential not only because the size of the problems can be large, but also in real applications the problems have to be solved in real-time. Moreover, In choosing optimal system parameters, one has to solve the above large system of linear equations for a large number of times. Hence a reduction in computational time will be crucial.

\section{Properties of the Generator Matrix}

In this section, we explore the near-Toeplitz structure of the generator matrix $\mathbf{H}_{n}$. In fact by modifying the entries of the first row, the last row and the first $s$ entries of the main diagonal of $\mathbf{H}_{n}$, one can turn the generator matrix into a Toeplitz matrix. The matrix $\mathbf{H}_{n}$ can be written as the sum of a Toeplitz matrix $\mathbf{T}_{n}$ and a low rank matrix, the same is the matrix $\mathbf{Q}_{n}$, i.e.,

$$
\mathbf{Q}_{n}=\mathbf{T}_{n}+\mathbf{R}_{n}
$$

This property is important in developing fast numerical algorithm for solving the steady-state probability distribution. We call a matrix as Toeplitz matrix if it has constant diagonals, i.e.

$$
\mathbf{T}_{n}=\left(\begin{array}{cccccc}
t_{0} & t_{-1} & t_{-2} & \cdots & t_{2-n} & t_{2-n} \\
t_{1} & t_{0} & t_{-1} & \cdots & \cdots & t_{1-n} \\
\vdots & \ddots & \ddots & \ddots & \ddots & \vdots \\
\vdots & \ddots & \ddots & \ddots & \ddots & \vdots \\
t_{n-2} & \cdots & \cdots & \ddots & \ddots & t_{-1} \\
t_{n-1} & t_{n-2} & \cdots & \cdots & t_{1} & t_{0}
\end{array}\right)
$$


In general, the sequence of $n \times n$ Toeplitz matrices is generated by a function $f(\theta) \in[-\pi, \pi]$ in Wiener class(This means it has a Fourier series with absolutely summable Fourier coefficient). We let $\mathcal{T}_{n}[f]$ to be the $n \times n$ Toeplitz matrix with $(j, k)$-th entry given by the $(j-k)$ th Fourier coefficient of $f(\theta)$, i.e.,

$$
t_{k}=\frac{1}{2 \pi} \int_{-\pi}^{\pi} f(\theta) e^{-i k \theta} d \theta, \quad k=0, \pm 1, \pm 2, \ldots
$$

If the Toeplitz matrix $\mathbf{T}_{n}$ is generated by the function $f(\theta)$, we denote as $\mathbf{T}_{n}=\mathcal{T}_{n}[f]$.

\section{The Gohberg-Semumcul Formula}

In this section, we introduce a useful formula for computing the inverse of a Toeplitz matrix. We denote $\mathbf{e}_{n}^{(k)}$ be the $n \times 1$ unit vectors, where the subscript denotes the length of the vector and the superscript denotes the position of the nonzero element. Let

$$
\mathbf{Z}_{n}=\left(\mathbf{e}_{n}^{(2)}, \mathbf{e}_{n}^{(3)}, \ldots, \mathbf{e}_{n}^{(n)}, \mathbf{0}\right) \text { and } \mathbf{J}_{n}=\left(\mathbf{e}_{n}^{(n)}, \mathbf{e}_{n}^{(n-1)}, \ldots, \mathbf{e}_{n}^{(2)}, \mathbf{e}_{n}^{(1)}\right) .
$$

then for any $n \times 1$ vector $\mathbf{a}=\left(a_{1}, a_{2}, \ldots, a_{n}\right)^{t}$, we use the vectors $\overline{\mathbf{a}}$ and $\underline{\mathbf{a}}$ to denote $\mathbf{Z}_{n} \mathbf{a}$ and $\mathbf{Z}_{n}^{t} \mathbf{a}$ respectively, i.e.,

$$
\overline{\mathbf{a}}=\left(0, a_{1}, a_{2}, \ldots, a_{n-1}\right)^{t} \quad \text { and } \quad \underline{\mathbf{a}}=\left(a_{2}, a_{3}, \ldots, a_{n}, 0\right)^{t} .
$$

We denote $\mathbf{L}(\mathbf{a})$ as the lower-triangular Toeplitz matrix with its first column entries being given by a with its first row being given by $\left(a_{1}, a_{2}, \ldots, a_{n}\right)^{t}$, and $\mathbf{U}(\mathbf{a})$ as the upper-triangular Toeplitz matrix with its first row being given by $\left(a_{n}, a_{n-1}, \ldots, a_{1}\right)^{t}$, i.e.,

$$
\mathbf{L}(\mathbf{a})=\left(\begin{array}{cccc}
a_{1} & & & 0 \\
a_{2} & a_{1} & & \\
\vdots & \ddots & \ddots & \\
a_{n} & \cdots & a_{2} & a_{1}
\end{array}\right) \quad \text { and } \quad \mathbf{U}(\mathbf{a})=\left(\begin{array}{cccc}
a_{n} & a_{n-1} & \cdots & a_{1} \\
& \ddots & \ddots & \vdots \\
& & a_{n} & a_{n-1} \\
0 & & & a_{n}
\end{array}\right)
$$

Let $\mathbf{x}_{n}=\left(x_{1}, x_{2}, \ldots, x_{n}\right)^{t}$ and $\mathbf{y}_{n}=\left(y_{1}, y_{2}, \ldots, y_{n}\right)^{t}$ be the solutions of the linear systems

$$
\begin{aligned}
& \mathbf{T}_{n} \mathbf{x}_{n}=\mathbf{e}_{n}^{(1)} \\
& \mathbf{T}_{n} \mathbf{y}_{n}=\mathbf{e}_{n}^{(n)} .
\end{aligned}
$$

It is well-known that the inverse of a Toeplitz matrix can be written as a sum of multiplications of lower-triangular and upper-triangular Toeplitz matrices [7]. If the linear system (6) is solvable, then the inverse of $T_{n}$ can be constructed by using the famous Gohberg-Semumcul formula [7].

For any positive definite Toeplitz matrix $\mathbf{T}_{n}$, if $\mathbf{x}_{n}$ and $\mathbf{y}_{n}$ are the solutions of (6) and (7), we must have $x_{1} \neq 0$, see for instance [9] and the inverse of $\mathbf{T}_{n}$ can be represented in terms of $\mathbf{x}_{n}$ and $\mathbf{y}_{n}$ by using Gohberg-Semumcul formula [7]:

$$
\mathbf{T}_{n}^{-1}=\frac{1}{x_{1}}\left[\mathbf{L}\left(\mathbf{x}_{n}\right) \mathbf{U}\left(\mathbf{y}_{n}\right)-\mathbf{L}\left(\overline{\mathbf{y}}_{n}\right) \mathbf{U}\left(\underline{\mathbf{x}}_{n}\right)\right] .
$$


When $x_{1}$ is zero, Ben-Artzi and Shalom [1], Labahn and Shalom [11], Ng, Rost and Wen [13] and Heinig [9] have studied the representation. Furthermore, if the nonsingular matrix $T_{n}$ is well-conditioned, Gohberg-Semumcul formula is numerically forward stable, Wen et al. [15].

The following lemma gives the relationships between the solutions of (6) and (7) from the size of $n$ to the size of $2 n$.

Lemma 1 [16] Let $\mathbf{T}_{n}=\left(t_{i-j}\right)_{i j}$ be a non-singular Toeplitz matrix and we denote

$$
\mathbf{c}_{n}=\left(t_{0}, t_{1}, \ldots, t_{n-1}\right)^{t} \quad \text { and } \quad \mathbf{r}_{n}=\left(t_{0}, t_{-1}, \ldots, t_{1-n}\right)^{t} .
$$

Suppose that $\mathbf{x}_{n}$ and $\mathbf{y}_{n}$ satisfy the equations (6) and (7) respectively and $x_{1} \neq 0$ then the following equations are solvable:

$$
\mathbf{L}\left(\mathbf{x}_{n}\right) \mathbf{u}_{n}=-\mathbf{U}\left(\underline{\mathbf{c}_{n}}\right) \mathbf{x}_{n} \text { and } \mathbf{U}\left(\mathbf{y}_{n}\right) \mathbf{v}_{n}=-\mathbf{L}\left(\mathbf{J}_{n} \underline{\mathbf{r}_{n}}\right) \mathbf{y}_{n}
$$

Here

$$
\mathbf{u}_{n}=\left(u_{1}, u_{2}, \ldots, u_{n}\right)^{t} \quad \text { and } \quad \mathbf{v}_{n}=\left(v_{1}, v_{2}, \ldots, v_{n}\right)^{t} .
$$

Moreover, if we define $\mathbf{P}_{2 n}$ as

$$
\mathbf{P}_{2 n}=\left(\begin{array}{ll}
\mathbf{P}_{2 n}^{1,1} & \mathbf{P}_{2 n}^{1,2} \\
\mathbf{P}_{2 n}^{2,1} & \mathbf{P}_{2 n}^{2,2}
\end{array}\right)
$$

with

$$
\mathbf{P}_{2 n}^{1,1}=\mathbf{P}_{2 n}^{2,2}=\mathbf{T}_{n}, \quad \mathbf{P}_{2 n}^{2,1}=\mathbf{L}\left(\mathbf{u}_{n}\right)+\mathbf{U}\left(\underline{\mathbf{c}_{n}}\right) \quad \text { and } \quad \mathbf{P}_{2 n}^{1,2}=\mathbf{L}\left(\mathbf{J}_{n} \underline{\mathbf{r}_{n}}\right)+\mathbf{U}\left(\mathbf{v}_{n}\right)
$$

then we have

$$
\begin{gathered}
\mathbf{P}_{2 n} \mathbf{x}_{2 n}^{(P)}=\mathbf{e}_{2 n}^{(1)} \text { and } \mathbf{P}_{2 n} \mathbf{y}_{2 n}^{(P)}=\mathbf{e}_{2 n}^{(2 n)} \\
\text { Here } \mathbf{x}_{2 n}^{(P)}=\left(\begin{array}{c}
\mathbf{x}_{n} \\
0
\end{array}\right) \text { and } \mathbf{y}_{2 n}^{(P)}=\left(\begin{array}{c}
0 \\
\mathbf{y}_{n}
\end{array}\right)
\end{gathered}
$$

If the Toeplitz matrix $\mathbf{T}_{2 n}$ is generated by the function $f(\theta)$, we denote the matrix $\mathbf{P}_{2 n}$ obtained by Lemma 1 as $\mathcal{P}_{2 n}[f]$. We remark it is not necessary to obtain the solutions $\mathbf{u}_{n}$ and $\mathbf{v}_{n}$ using (9) in order to construct the inverse of $\mathbf{P}_{2 n}$. In fact, we have the following formula:

$$
\mathbf{P}_{2 n}^{-1}=\frac{1}{x_{1}}\left[\mathbf{L}\left(\mathbf{x}_{2 n}^{(P)}\right) \mathbf{U}\left(\mathbf{y}_{2 n}^{(P)}\right)-\mathbf{L}\left(\overline{\mathbf{x}}_{2 n}^{(P)}\right) \mathbf{U}\left(\underline{\mathbf{y}}_{2 n}^{(P)}\right)\right]
$$

\section{Construction of Preconditioner and Convergence Anal- ysis}

In this section, we construct an approximate inverse-free preconditioner for Toeplitz system. We need the following definitions before our discussions. 


\subsection{On the Diagonal-off Decay of the Matrices}

Definition 1 [12] Let $\mathbf{A}=\left[a_{i, j}\right]_{i, j \in I}$ be a matrix, where the index set $I$ can be $\mathbf{Z}$, $\mathbf{N}$, or $\{1,2, \ldots, N\}$.

1. A belongs to the space $\mathcal{E}_{\gamma}$ if

$$
\left|a_{i, j}\right| \leq c e^{-\gamma|i-j|} \quad \text { for } \quad \gamma>0
$$

and some constant $c>0$.

2. A belongs to the space $\mathcal{Q}_{\eta}$ if

$$
\left|a_{i, j}\right| \leq c(1+|i-j|)^{-\eta} \quad \text { for } \quad \eta>1,
$$

and some constant $c>0$.

With these definitions, we have the following results about the off-diagonal decay of the entries of $\mathbf{A}^{-1}$.

Theorem 1 [10] Let $\mathbf{A}: l^{2}(I) \rightarrow l^{2}(I)$ be an invertible matrix, where $I=\mathbf{Z}, \mathbf{N}$ or $\{1,2, \ldots, N\}$.

1. If $\mathbf{A} \in \mathcal{E}_{\gamma}$, then $\mathbf{A}^{-1} \in \mathcal{E}_{\gamma_{1}}$ for some $\gamma_{1} \in(0, \gamma)$.

2. If $\mathbf{A} \in \mathcal{Q}_{\eta}$, then $\mathbf{A}^{-1} \in \mathcal{Q}_{\eta}$.

Lemma 2 Let $t_{j}(j=0,1,2, \ldots)$ be a sequence with its entries satisfying (13) or (14) for some $c>0$. Then the sequence $\left\{t_{j}\right\}$ is absolutely summable, i.e., $\sum_{k=0}^{\infty}\left|t_{j}\right|$ is bounded, and for any given $\epsilon>0$, there exists a constant $K>0$ independent of $n$, such that for all $n>K$,

$$
\sum_{k=K}^{n}\left|t_{j}\right|<\epsilon .
$$

We note that there is a natural partitioning of a Toeplitz matrix into $2 \times 2$ blocks as follows:

$$
\mathbf{T}_{2 n}=\left(\begin{array}{cc}
\mathbf{T}_{2 n}^{1,1} & \mathbf{T}_{2 n}^{1,2} \\
\mathbf{T}_{2 n}^{2,1} & \mathbf{T}_{2 n}^{2,2}
\end{array}\right)
$$

where $\mathbf{T}_{2 n}^{1,1}=\mathbf{T}_{2 n}^{2,2}=\mathbf{T}_{n}$ are principal submatrices of $\mathbf{T}_{2 n}, \mathbf{T}_{2 n}^{1,2}$ and $\mathbf{T}_{2 n}^{2,2}$ are also $n \times n$ Toeplitz matricies. We propose to use $\mathbf{P}_{2 n}$ defined in (10) as a preconditioner for $\mathbf{T}_{2 n}$.

Lemma 3 Let an $2 n \times 2 n$ positive definite Toeplitz matrix $\mathbf{T}_{2 n}$ generated by a strictly positive absolute value function $f(\theta)$ with its diagonal entries satisfying (13) or (14) for some $c>0$. Then for any given $\epsilon_{0}>0$, there exists a constant $K>0$ independent of $n$, such that for all $n>K$,

$$
\left\|\mathbf{P}_{2 n}^{2,1}-\mathbf{T}_{2 n}^{2,1}\right\|_{1}<\epsilon_{0} \quad \text { and } \quad\left\|\mathbf{P}_{2 n}^{2,1}-\mathbf{T}_{2 n}^{2,1}\right\|_{\infty}<\epsilon_{0} .
$$

and

$$
\left\|\mathbf{P}_{2 n}^{1,2}-\mathbf{T}_{2 n}^{1,2}\right\|_{1}<\epsilon_{0} \quad \text { and } \quad\left\|\mathbf{P}_{2 n}^{1,2}-\mathbf{T}_{2 n}^{1,2}\right\|_{\infty}<\epsilon_{0} .
$$

Here $\mathbf{P}_{2 n}^{2,1}$ and $\mathbf{P}_{2 n}^{1,2}$ defined in 10 and $\mathbf{T}_{2 n}^{2,1}$ and $\mathbf{T}_{2 n}^{1,2}$ defined in (16). 
Theorem 2 Let an $2 n \times 2 n$ positive definite Toeplitz matrix $\mathbf{T}_{2 n}$ generated by a strictly positive absolute value function $f(\theta)$ with its diagonal entries satisfying (13) or (14) for some $c>0$. Then for any given $\epsilon_{0}>0$, there exists a constant $K>0$ independent of $n$, such that for all $n>K$,

$$
\left\|\mathbf{T}_{2 n}-\mathbf{P}_{2 n}\right\|_{2}<\epsilon_{0} .
$$

Here $\mathbf{P}_{2 n}$ defined in (10). Moreover, for any given $\epsilon_{1}>0$, there exists a constant $K_{1}>0$ such that for all $n>K_{1}$,

$$
\left\|\mathbf{P}_{2 n}^{-1}-\mathbf{T}_{2 n}^{-1}\right\|_{2}<\epsilon_{1}
$$

Now we consider the more interesting case that the generating function $f(\theta)$ to have zeros. Suppose that the generating function $f(\theta) \in C_{2 \pi}$ be non-negative and have finite zeros. Let $\theta_{k}(k=1,2, \ldots, m)$ be all roots of $f(\theta)$ in $[-\pi, \pi)$ with order $a_{j}$. One can write

$$
f(\theta)=h(\theta) w(\theta), \quad-\pi \leq \theta<\pi
$$

where $w(\theta)=\prod_{k=1}^{m}\left(1-\cos \left(\theta-\theta_{k}\right)\right)^{a_{k}}$ and $|h(\theta)|>0$. It is easy to see that $\mathcal{T}_{n}[w]$ is a unit lower triangular Toeplitz matrix with bandwidth $a=\sum_{k=1}^{m} a_{k}$. By straightforward calculation, we obtain

$$
\mathcal{T}_{n}[f]=\mathcal{T}_{n}[w] \mathcal{T}_{n}[h]+\mathbf{G}_{n}
$$

where $\mathbf{G}_{n}$ has only non-zeros entries in its first $a$ columns and therefore its rank is less than $a$.

Theorem 3 Let an $2 n \times 2 n$ positive definite Toeplitz matrix $\mathbf{T}_{2 n}$ be generated by $f(\theta)$ in (21) with its diagonal entries satisfying (13) or (14) for some $c>0$. Then for any given $\epsilon_{0}>0$, there exists a constant $K>0$ independent of $n$, such that for all $n>K$, at most a eigenvalues of

$$
\mathcal{T}_{2 n}[f]-\mathcal{T}_{2 n}[w] \mathcal{P}_{2 n}[h]
$$

have absolute value exceeding $\epsilon_{0}$.

We recall that the matrix $\mathbf{Q}_{n}$ in the queueing problem can be written as the sum of a Toeplitz matrix $\mathbf{T}_{n}$ with the generating function $f(\theta)$ and a matrix with rank $s+1$. Also the function $f(\theta)$ can be written the form in (22). Denote $\mathbf{P}_{2 n}=\mathcal{P}_{2 n}[h]^{-1} \mathcal{T}[w]^{-1}$, using the above theorem, we show that $\mathbf{P}_{2 n}^{-1} \mathbf{Q}_{2 n}$ can be written as $\mathbf{P}_{2 n}^{-1} \mathbf{Q}_{2 n}=\mathbf{I}_{2 n}+\mathbf{F}_{2 n}+\mathbf{K}_{2 n}$, where $\mathbf{I}_{2 n}$ is the identity matrix, $\mathbf{F}_{2 n}$ is a matrix with small 2-norm, and $\mathbf{K}_{2 n}$ is a matrix with small rank $s+a+1$. We have the following theorem.

Theorem 4 Let $\mathbf{Q}_{2 n}$ be the matrix in 3, and $\mathbf{T}_{2 n}$ is generated by $f(\theta)$ in (21) with its diagonal entries satisfying (13) or (14) for some $c>0$. We denote $\mathbf{P}_{2 n}=\mathcal{P}_{2 n}[h]^{-1} \mathcal{T}[w]^{-1}$. Then for any given $\epsilon_{0}>0$, there exists a constant $K>0$ independent of $n$, such that for all $n>K$, at most $s+a+1$ eigenvalues of $\mathbf{P}_{2 n}^{-1} \mathbf{Q}_{2 n}$ have absolute value exceeding $\epsilon_{0}$. 


\section{$5 \quad$ Numerical Results}

In this section, we apply GMRES algorithm to solve the equation (2) using $\mathbf{P}_{n}$ as a precondtioner. The generating function $f(\theta)$ of the Toeplitz matrix in (3) is implicit in practical, we remark it is not necessary to construct $f(\theta)$ first, our scheme is to solve the equation (6) and (7) and obtain the solution $\mathbf{x}_{n / 2}$ and $\mathbf{y}_{n / 2}$, then $\mathbf{P}_{n}^{-1}$ can be constructed by (12). The solution of $\mathbf{x}_{n / 2}$ and $\mathbf{y}_{n / 2}$ involved in the preconditioner $\mathbf{P}_{n}^{-1}$ can be recursively generated by solving (6) and (7) until the size of the linear system is sufficiently small. The algorithm is described in Table 1. The total cost of the recursive computation is roughly bounded by $O(n \log n)$.

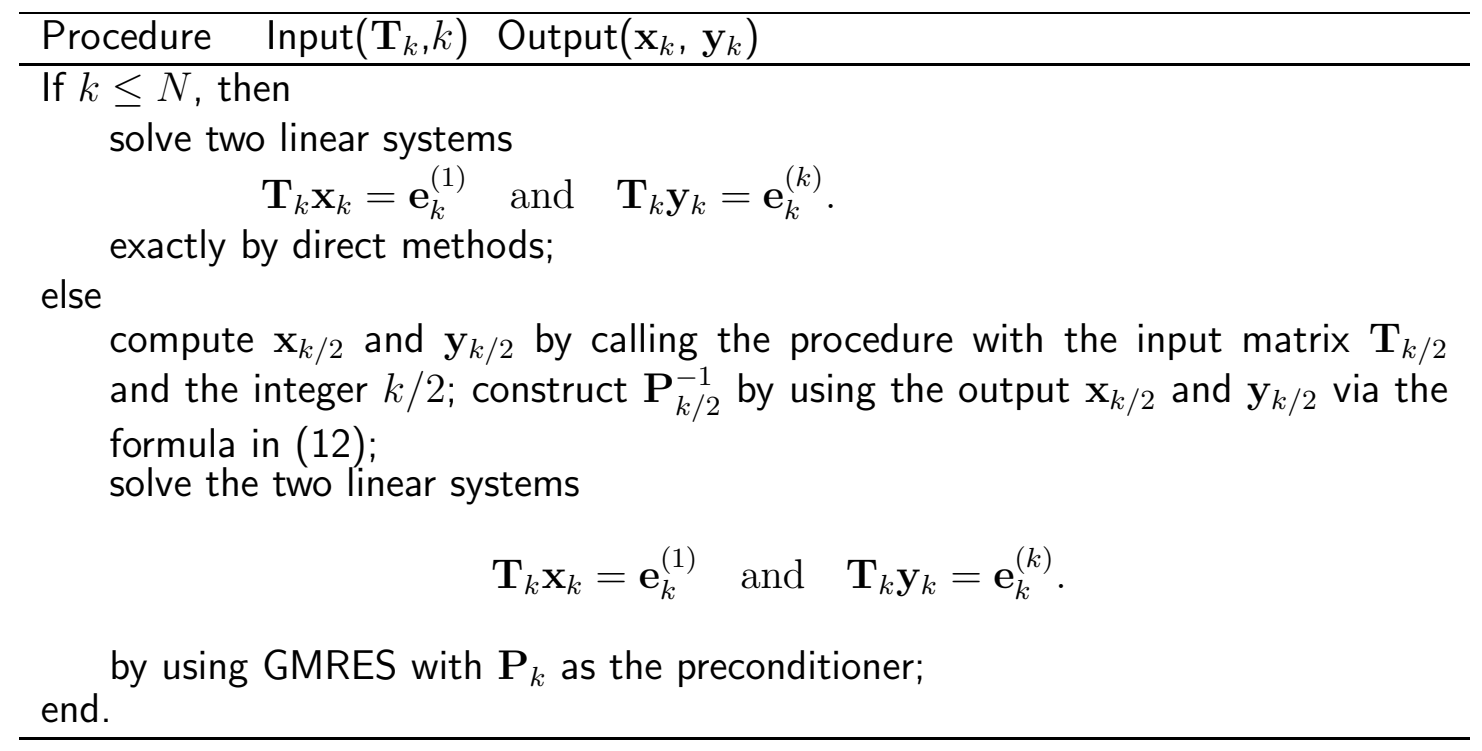

Table 1: Recursive Algorithm to obtain $\mathbf{x}_{n / 2}$ and $\mathbf{y}_{n / 2}$ in order to construct the preconditioner $\operatorname{matrix} \mathbf{P}_{n}$.

We show our numerical results for two sets of queueing parameters which is tested in [2]:

$$
\begin{aligned}
& \text { 1. } \lambda_{j}=\frac{1}{2^{j-1}}, j=1,2, \ldots \text {, and } \tau_{j}=\frac{0.7744}{j^{j}}, j=1,2, \ldots . \\
& \text { 2. } \lambda_{j}=\frac{1}{2^{j-1}}, j=1,2, \ldots \text {, and } \tau_{j}=\frac{90}{(\pi j)^{4}}, j=1,2, \ldots
\end{aligned}
$$

For each case, we tried three different choices of number of servers $s: s=1,2$ and 4 . The service rate $\mu$ is set to $\mu=1$. Also, we note that in both cases

$$
\lambda=\sum_{k=1}^{\infty} \lambda_{k}=2 \quad \text { and } \quad \tau=\sum_{k=1}^{\infty} \tau_{k}=1 .
$$

The initial guess for both methods is the unit vector $(0, \ldots, 0,1)^{t}$. The stopping criterion for all the methods is

$$
\frac{\left\|\mathbf{r}_{k}\right\|_{2}}{\left\|\mathbf{r}_{0}\right\|_{2}}<10^{-6}
$$


Table 2: Number of iterations for $\lambda_{j}=\frac{1}{2^{j-1}}$ and $\tau_{j}=\frac{0.7744}{j^{j}}$.

\begin{tabular}{c|ccc|ccc|cccc}
\hline & \multicolumn{4}{|c|}{$s=1$} & \multicolumn{4}{c|}{$s=2$} & \multicolumn{3}{c}{$s=4$} \\
\cline { 2 - 10 }$n$ & $\mathbf{I}_{n}$ & $\mathbf{P}_{n}$ & $\widetilde{\mathbf{P}}_{n}$ in $[2]$ & $\mathbf{I}_{n}$ & $\mathbf{P}_{n}$ & $\widetilde{\mathbf{P}}_{n}$ in $[2]$ & $\mathbf{I}_{n}$ & $\mathbf{P}_{n}$ & $\widetilde{\mathbf{P}}_{n}$ in $[2]$ \\
\hline 32 & 31 & 7 & 8 & 32 & 10 & 9 & 32 & 11 & 10 \\
64 & 36 & 5 & 8 & 64 & 8 & 10 & 64 & 11 & 11 \\
128 & 116 & 5 & 8 & 104 & 6 & 10 & 128 & 11 & 11 \\
256 & 227 & 5 & 8 & 146 & 5 & 10 & 253 & 8 & 12 \\
512 & 447 & 5 & 8 & 294 & 5 & 10 & 491 & 8 & 12 \\
1024 & $* *$ & 5 & 8 & $* *$ & 5 & 10 & $* *$ & 8 & 12 \\
2048 & $* *$ & 5 & 8 & $* *$ & 5 & 10 & $* *$ & 8 & 12 \\
4096 & $* *$ & 5 & 8 & $* *$ & 5 & 10 & $* *$ & 8 & 12 \\
\hline
\end{tabular}

Table 3: Number of iterations for $\lambda_{j}=\frac{1}{2^{j-1}}$ and $\tau_{j}=\frac{90}{(\pi j)^{4}}$.

\begin{tabular}{c|ccc|ccc|cccc}
\hline \multirow{2}{*}{$n$} & \multicolumn{3}{|c|}{$s=1$} & \multicolumn{3}{c|}{$s=2$} & \multicolumn{3}{c}{$s=4$} \\
\cline { 2 - 10 } & $\mathbf{I}_{n}$ & $\mathbf{P}_{n}$ & $\mathbf{P}_{n}$ in $[2]$ & $\mathbf{I}_{n}$ & $\mathbf{P}_{n}$ & $\mathbf{P}_{n}$ in $[2]$ & $\mathbf{I}_{n}$ & $\mathbf{P}_{n}$ & $\mathbf{P}_{n}$ in $[2]$ \\
\hline 32 & 32 & 5 & 7 & 32 & 8 & 8 & 32 & 11 & 9 \\
64 & 35 & 4 & 7 & 64 & 7 & 8 & 64 & 11 & 9 \\
128 & 37 & 3 & 7 & 92 & 4 & 8 & 128 & 10 & 9 \\
256 & 37 & 2 & 7 & 92 & 4 & 8 & 256 & 9 & 9 \\
512 & 37 & 2 & 7 & 92 & 3 & 8 & $* *$ & 8 & 9 \\
1024 & 37 & 2 & 7 & 92 & 2 & 8 & $* *$ & 8 & 9 \\
2048 & 37 & 2 & 7 & 92 & 2 & 8 & $* *$ & 8 & 9 \\
4096 & 37 & 2 & 7 & 92 & 2 & 8 & $* *$ & 8 & 9 \\
\hline
\end{tabular}

where $\mathbf{r}_{k}$ is the residual at the kth iteration.

We use Matlab to test our algorithm on the above examples. The tables (2) and (3) give the number of iterations required for convergence by using different methods and preconditioners. Here $\mathbf{I}_{n}, \mathbf{P}_{n}$ and $\widetilde{\mathbf{P}}_{n}$ mean the GMRES method is used without preconditioner, with our preconditioner and with preconditioner in [2] respectively. The symbol "**" means that the method does not converge in 500 iterations. According to Tables (2) and (3), we see that the number of iterations for the non-preconditioned systems (column " $\mathbf{I}_{n}$ ") increases when the size $n$ increases. However, the number of iterations for the preconditioner $\widetilde{\mathbf{P}}_{n}$ increases or almost keep constant when the size $n$ increases. While the number of iterations for our preconditioner $\mathbf{P}_{n}$ decreases or almost keep constant. Numerical results show that our preconditioner is very efficient. 


\section{Appendix}

\subsection{Proof of Lemma 2}

Proof: At first, we consider the case $\left|t_{j}\right| \leq c e^{-\gamma|j|}$, we have

$$
\sum_{k=p}^{n}\left|t_{j}\right| \leq \sum_{k=p}^{n} c e^{-\gamma j}=\frac{1}{1-e^{-\gamma}} c e^{-\gamma p}\left(1-e^{-\gamma(n-p+1)}\right)<\frac{c e^{-\gamma p}}{1-e^{-\gamma}}
$$

Therefore, let $p=0$, we obtain $\left\{t_{j}\right\}$ is absolutely summable. Let

$$
K>-\gamma^{-1} \ln \left(c^{-1}\left(1-e^{-\gamma}\right) \epsilon\right)
$$

we obtain (15).

When $\left|t_{j}\right| \leq c(|j|+1)^{-\eta}$, we have

$$
\sum_{k=p}^{n}\left|t_{j}\right| \leq \sum_{k=p}^{n} c(|j|+1)^{-\eta} \leq \sum_{k=p}^{\infty} c(|j|+1)^{-\eta} \leq c \int_{p-1}^{\infty}(x+1)^{-\eta} d x \leq \frac{c p^{1-\eta}}{\eta-1}
$$

We know $\left\{t_{j}\right\}$ is absolutely summable. Let

$$
K>\left(\frac{c}{(\eta-1) \epsilon}\right)^{1 /(\eta-1)}
$$

then (15) holds.

\subsection{Proof of Lemma 3}

Proof: We only consider the case $\mathbf{T}_{2 n} \in \mathcal{E}_{\gamma}$, i.e. $\left|t_{j}\right| \leq c e^{-\gamma|j|}$, the other case can be similar. Notice that $\mathbf{x}_{n}$ is the first column of $\mathbf{T}_{n}$, we deduce that

$$
\mathbf{L}\left[\mathbf{x}_{n}\right] \in \mathcal{E}_{\gamma_{1}}
$$

and

$$
x_{n, k} \leq c_{1} e^{-\gamma_{1} k}
$$

with $0<\gamma_{1} \leq \gamma$,

$$
\mathbf{L}\left[\mathbf{x}_{n}\right]^{-1} \in \mathcal{E}_{\gamma_{2}}
$$

and

$$
\left(\mathbf{L}\left[\mathbf{x}_{n}\right]^{-1}\right)_{j, k} \leq c_{2} e^{-\gamma_{2}|j-k|}
$$

with $0<\gamma_{2} \leq \gamma_{1}$. From Lemma 2, there exist two constants $M_{1}, M_{2}, M_{3}$ independent of $n$, such that

$$
\sum_{k=0}^{2 n-1}\left|t_{k}\right|<M_{1}, \quad \sum_{k=1}^{n}\left|x_{n}\right|<M_{2} \quad \text { and } \quad\left\|\mathbf{L}\left[\mathbf{x}_{n}\right]^{-1}\right\|_{1} \leq M_{3}
$$


Thus, for any given $\varepsilon$, there exist constant $N_{1}$, such that for all $n>N_{1}$,

$$
\sum_{k=N_{1}}^{2 n-1}\left|t_{k}\right|<\varepsilon .
$$

Also there exist constant $N_{2}$, such that for all $n>N_{2}$,

$$
\sum_{k=N_{2}}^{n}\left|x_{n}\right|<\varepsilon .
$$

We denote

$$
\widetilde{\mathbf{x}}_{n}=\left(0, \ldots, 0, x_{n-N_{1}+2}, x_{n-N_{1}+3}, \ldots, x_{n}\right)^{t}
$$

and

$$
\mathbf{E}_{1}=\left(\begin{array}{cc}
0 & \widehat{\mathbf{E}}_{1} \\
0 & 0
\end{array}\right)
$$

with

$$
\widehat{\mathbf{E}}_{1}=\left(\begin{array}{cccc}
t_{N_{1}} & t_{N_{1}-1} & \cdots & t_{2} \\
& t_{N_{1}} & \ddots & \vdots \\
& & \ddots & t_{N_{1}-1} \\
0 & & & t_{N_{1}}
\end{array}\right) .
$$

For the above analysis, if $n>N_{1}+N_{2}$, we have

$$
\left\|\mathbf{U}\left[\underline{\mathbf{c}_{n}}\right]-\mathbf{E}_{1}\right\|_{1}<\varepsilon, \quad\left\|\mathbf{E}_{1}\right\|_{1}<M_{1}, \quad\left\|\widetilde{\mathbf{x}}_{n}\right\|_{1}<\varepsilon \quad \text { and } \quad\left\|\mathbf{x}_{n}\right\|_{1}<M_{2} .
$$

Thus, using $\mathbf{E}_{1} \mathbf{x}_{n}=\mathbf{E}_{1} \widetilde{\mathbf{x}}_{n}$, we have

$$
\begin{aligned}
\left\|\mathbf{U}\left[\underline{\mathbf{c}_{n}}\right] \mathbf{x}_{n}\right\|_{1} & =\|\left(\mathbf{U}\left[\underline{\left.\mathbf{c}_{n}\right]}-\mathbf{E}_{1}\right) \mathbf{x}_{n}+\mathbf{E}_{1} \widetilde{\mathbf{x}}_{n}\left\|_{1} \leq\right\|\left(\mathbf{U}\left[\underline{\mathbf{c}_{n}}\right]-\mathbf{E}_{1}\right) \mathbf{x}_{n}+E_{1} \widetilde{\mathbf{x}}_{n} \|_{1}\right. \\
& \leq\left\|\left(\mathbf{U}\left[\underline{\mathbf{c}_{n}}\right]-\mathbf{E}_{1}\right)\right\|_{1}\left\|\mathbf{x}_{n}\right\|_{1}+\left\|\mathbf{E}_{1}\right\|_{1}\left\|\widetilde{\mathbf{x}}_{n}\right\|_{1}<\left(M_{1}+M_{2}\right) \varepsilon .
\end{aligned}
$$

Thus we obtain

$$
\left\|\mathbf{u}_{n}\right\|_{1}=\left\|-\mathbf{L}\left(\mathbf{x}_{n}\right)^{-1} \mathbf{U}\left[\underline{\mathbf{c}_{n}}\right] \mathbf{x}_{n}\right\|_{1} \leq\left\|L\left(\mathbf{x}_{n}\right)^{-1}\right\|_{1}\left\|\mathbf{U}\left[\underline{\mathbf{c}_{n}}\right] \mathbf{x}_{n}\right\|_{1} \leq M_{3}\left(M_{1}+M_{2}\right) \varepsilon .
$$

Denote

$$
\mathbf{t}_{2}=\left(t_{n}, t_{n+1}, \ldots, t_{2 n-1}\right)^{t}
$$

then we have

$$
\left\|\mathbf{P}_{2 n}^{2,1}-\mathbf{T}_{2 n}^{2,1}\right\|_{1}=\left\|\mathbf{L}\left[\mathbf{t}_{2}\right]-\mathbf{L}\left[\mathbf{u}_{n}\right]\right\|_{1} \leq\left\|\mathbf{t}_{2}\right\|_{1}+\left\|\mathbf{u}_{n}\right\|_{1} \leq \varepsilon+M_{3}\left(M_{1}+M_{2}\right) \varepsilon .
$$

By letting

$$
\varepsilon_{0}=\frac{1}{M\left(M_{1}+M_{2}\right)+x_{1}} \epsilon
$$


(17) holds. Similarly, we obtain

$$
\left\|\mathbf{P}_{2 n}^{2,1}-\mathbf{T}_{2 n}^{2,1}\right\|_{\infty}<\varepsilon_{0}
$$

We note that the structure of

$$
\mathbf{J}_{n}\left(\mathbf{P}_{2 n}^{1,2}-\mathbf{T}_{2 n}^{1,2}\right) \mathbf{J}_{n}
$$

is similar to that of $\mathbf{P}_{2 n}^{2,1}-\mathbf{T}_{2 n}^{2,1}$ and

$$
\mathbf{J}_{n}\left(\mathbf{P}_{2 n}^{1,2}-\mathbf{T}_{2 n}^{1,2}\right) \mathbf{J}_{n}=\left(\mathbf{P}_{2 n}^{1,2}-\mathbf{T}_{2 n}^{1,2}\right)^{t} .
$$

Therefore the proof of (18) is very similar to the proof of (17).

\subsection{Proof of Theorem 2}

Proof: Denote $\mathbf{E}=\mathbf{T}_{2 n}-\mathbf{P}_{2 n}$, we have

$$
\mathbf{E}=\mathbf{T}_{2 n}-\mathbf{P}_{2 n}=\left(\begin{array}{cc}
0 & \mathbf{P}_{2 n}^{1,2}-\mathbf{T}_{2 n}^{1,2} \\
\mathbf{P}_{2 n}^{2,1}-\mathbf{T}_{2 n}^{2,1} & 0
\end{array}\right) .
$$

where $\mathbf{P}_{2 n}^{2,1}$ and $\mathbf{P}_{2 n}^{1,2}$ defined in (10) and $\mathbf{T}_{2 n}^{2,1}$ and $\mathbf{T}_{2 n}^{1,2}$ defined in (16). According to Lemma (3), we get

$$
\|\mathbf{E}\|_{1}=\max \left\{\left\|\mathbf{P}_{2 n}^{2,1}-\mathbf{T}_{2 n}^{2,1}\right\|_{1},\left\|\mathbf{P}_{2 n}^{1,2}-\mathbf{T}_{2 n}^{1,2}\right\|_{1}\right\}<\epsilon_{0}
$$

and

$$
\|\mathbf{E}\|_{\infty}=\max \left\{\left\|\mathbf{P}_{2 n}^{2,1}-\mathbf{T}_{2 n}^{2,1}\right\|_{\infty},\left\|\mathbf{P}_{2 n}^{1,2}-\mathbf{T}_{2 n}^{1,2}\right\|_{\infty}\right\}<\epsilon_{0}
$$

Therefore, we obtain

$$
\|\mathbf{E}\|_{2} \leq \sqrt{\|\mathbf{E}\|_{1}\|\mathbf{E}\|_{\infty}}<\epsilon_{0} .
$$

We note that $\mathbf{T}_{2 n}^{-1}$ and $\mathbf{P}_{2 n}^{-1}$ is bounded, thus it is similarly to induce that (20) is also hold.

\subsection{Proof of 3}

Proof: Notice that $h(\theta)>0$, from Theorem 2 we know for any given $\epsilon>0$, there exists a constant $K>0$ independent of $n$, such that for all $n>K$, we have

$$
\left\|\mathcal{T}_{2 n}[h]-\mathcal{P}_{2 n}[h]\right\|_{2}<\epsilon .
$$

Let $\epsilon_{0}=\left\|\mathcal{T}_{2 n}[w]\right\|_{2} \epsilon$, we have

$$
\mathcal{T}_{2 n}[f]-\mathcal{T}_{2 n}[w] \mathcal{P}_{2 n}[h]=\mathcal{T}_{2 n}[w]\left(\mathcal{T}_{2 n}[h]-\mathcal{P}_{2 n}[h]\right)+\mathbf{G}_{2 n}
$$

where $\operatorname{rank}\left(\mathbf{G}_{2 n}\right) \leq a$ and

$$
\left\|\mathcal{T}_{2 n}[w]\left(\mathcal{T}_{2 n}[h]-\mathcal{P}_{2 n}[h]\right)\right\|_{2}<\epsilon_{0}
$$

This means that

$$
\mathcal{T}_{2 n}[f]-\mathcal{T}_{2 n}[w] \mathcal{P}_{2 n}[h]
$$

is the sum of a matrix with small 2-norm and a matrix with rank $a$. The result holds. 


\section{References}

[1] A. Ben-Artzi and T. Shalom, On Inversion of Toeplitz and Close to Toeplitz Matrices, Linear Algebra Appl., 75 (1986) 173-192.

[2] Ching, W., Iterative Methods for Queueing Systems with Batch Arrivals and Negative Customers, BIT, 43 (2003) 285-296.

[3] Ching W., Ng M.: Markov Chains : Models, Algorithms and Applications. International Series on Operations Research and Management Science. Springer: New York, (2006).

[4] E. Gelenbe, Random Neural Networks with Positive and Negative Signals and Product Solution, Neural Computation. 1 (1989), pp. 501-510.

[5] Gelenbe, P.Glynn, and K Sigman, Queues with Negative Arrivals, J. Appl. Prob. 28 (1991), pp. 245-250.

[6] E. Gelenbe, Product Form Networks with Negative and Positive Customers, J. Appl. Prob. 28 (1991), pp. 656-663.

[7] I. Gohberg and A. Semencul, On the Inversion of Finite Toeplitz Matrices and Their Continuous Analogs, Mat. Issled., 2 (1972) 201-233.

[8] P. Harrison, Reliability modeling Using G-queue, Euro. J. Oper. Res. 126 (2000), pp. 273387.

[9] G. Heinig, On the Reconstruction of Toeplitz Matrix Inverses from Columns, Linear Algebra Appl., 350 (2002) 199-212.

[10] S. Jaffard, Propriétés des matrices "bien localisées" près de leur diagonale et quelques applications, Ann. Inst. H. Poincaré Anal. Non Linéaire,, 7 (1990) 461-476.

[11] G. Labahn and T. Shalom, Inversion of Toeplitz Matrices with only Two Standard Equations, Linear Algebra Appl., 175 (1992) 143-158.

[12] T. Strohmer, Four Short Stories about Toeplitz Matrix Calculations, Linear Algebra Appl., 343-344 (2002) 321-344.

[13] M. Ng and K. Rost and Y. Wen, On Inversion of Toeplitz Matrices, Linear Algebra Appl., 348 (2002) 145-151.

[14] R. Varga, Matrix Iterative Analysis, Prentice-Hall, New Jersey, 1963.

[15] Y. Wen and M. Ng and W. Ching and H. Liu, A note on the Stability of Toeplitz Matrix Inversion Formulas, Appl. Math. Letters 17 (2004) 903-907.

[16] Y. Wen and W. Ching and M. Ng, Approximate Inverse-Free Preconditioners for Toeplitz Matrices, submitted. 